\title{
Oral versus patient-controlled intravenous administration of oxycodone for pain relief after cesarean section
}

\author{
Katja Mäkelä1,5通 Outi Palomäki ${ }^{1,5} \cdot$ Satu Pokkinen ${ }^{2,5} \cdot$ Arvi Yli-Hankala $^{2,5} \cdot$ Mika Helminen $^{3,4} \cdot$ Jukka Uotila $^{1,5}$
}

Received: 18 March 2019 / Accepted: 6 August 2019/ Published online: 17 August 2019

(c) The Author(s) 2019

\begin{abstract}
Purpose The optimal postoperative analgesia after cesarean section (CS) remains to be determined. The primary objective of this study was to assess whether oral oxycodone provides the same or better pain control and satisfaction with pain relief as oxycodone given intravenously using a patient-controlled analgesia (PCA) infusion device. The secondary objectives were to compare the gastrointestinal symptoms and postsurgical recovery of the two groups.

Methods This prospective randomized trial was conducted at a University Hospital between February 2015 and June 2017. Altogether 270 CS patients were randomly assigned to receive postoperative oxycodone pain relief by IV PCA $(n=133)$ or orally $(n=137)$. Pain control and satisfaction with pain treatment were assessed by a numeric rating scale (NRS) at $2,4,8$, and 24 h postoperatively.

Results No differences were found in NRS pain scores or satisfaction between the groups except at $24 \mathrm{~h}$ pain when coughing; there was a statistically significant difference favoring the IV PCA group $(p=0.006)$. In the IV PCA group, the patients experienced more nausea at $4 \mathrm{~h}(p=0.001)$ and more vomiting at $8 \mathrm{~h}(p=0.010)$. Otherwise, postoperative recovery was similar in both groups. The equianalgesic dose of oxycodone was significantly smaller in the oral group $(p=0.003)$.

Conclusions This study indicates that oral oxycodone provides pain control and satisfaction with pain relief equal to IV oxycodone PCA for postoperative analgesia after cesarean section. Satisfaction with pain treatment was high in both groups, and both methods were well tolerated. Early nausea was less common with oral medication.
\end{abstract}

Keywords Cesarean section $\cdot$ Oral analgesia $\cdot$ Oxycodone $\cdot$ Patient-controlled analgesia $\cdot$ Postoperative pain

$\begin{array}{ll}\text { Abbreviations } \\ \text { CS } & \text { Cesarean section } \\ \text { NRS } & \text { Numeric rating scale } \\ \text { PCA } & \text { Patient-controlled analgesia } \\ \text { NCA } & \text { Nurse-controlled analgesia } \\ \text { IV } & \text { Intravenous } \\ \text { IM } & \text { Intramuscular }\end{array}$

Katja Mäkelä

katja.makela@pshp.fi

1 Department of Obstetrics and Gynecology, Tampere University Hospital, PL 2000, 33521 Tampere, Finland

2 Department of Anesthesia, Tampere University Hospital, PL 2000, 33521 Tampere, Finland

3 Research, Development and Innovation Centre, Tampere University Hospital, PL 2000, 33521 Tampere, Finland

4 Faculty of Social Sciences, Health Sciences, Tampere University, ARVO, 33014 Tampere, Finland

5 Faculty of Medicine and Life Sciences, University of Tampere, Tampere, Finland
VAS Visual analogue scale

OX/NAL Extended-release oxycodone/naloxone tablet

\section{Introduction}

Cesarean section (CS) is one of the most common surgical operations in the world [1]. In some countries, the CS rate reaches over $40 \%[2,3]$. In Finland, the CS rate has remained low at about $16.7 \%$ [4]. Pain relief after CS is crucial, and it affects both the mother and child. Poor pain relief may adversely affect recovery, mother-infant bonding, and breast-feeding; it may even lead to persistent postsurgical pain. Pain after CS was ranked ninth for pain severity among 179 surgical procedures in a study of 115,775 patients [5]. There are variable methods to manage postoperative pain, and the search for the ideal method is still ongoing. The use of opioids via different routes is the gold standard, although several other techniques have also been proposed [6]. 
After CS, patients usually need opioid analgesia for 1-2 days. Intrathecal, epidural, intravenous (IV), intramuscular (IM), subcutaneous, or oral routes and a continuous or intermittent manner of dosing can be used [7]. In addition to opioids, patients are given anti-inflammatory analgesics to promote better pain relief. The results of studies comparing different methods for pain relief after surgical interventions are controversial [8].

Neuraxial or IV morphine is considered the standard choice of opioid for post-CS pain [9]. IV oxycodone has gained increasing popularity of late due to its faster onset of action, fewer or less severe adverse events, and better effects for visceral pain [10]. Oxycodone has good oral bioavailability and a longer duration of action compared to morphine, thus making it a good choice for per oral pain relief [11]. A recent review of oral oxycodone for acute postoperative surgery showed that when administered as part of a multimodal analgesic regimen, it produces superior pain relief with fewer side effects and lower costs compared to epidural and IV analgesics [12].

In our institution, both oral and IV patient-controlled analgesia (PCA)-based pain relief are used. Our primary goal was to investigate by randomized controlled trial whether orally administered oxycodone provides pain control that is equal to or better than IV PCA oxycodone in CS patients. Our secondary objectives were to compare gastrointestinal symptoms and postsurgical recovery between the groups.

\section{Materials and methods}

This prospective randomized study was conducted at the Department of Obstetrics and Gynaecology at Tampere University Hospital, Finland, between February 2015 and June 2017. During the study period, 1637 cesarean sections were conducted at the study hospital. The study protocol was approved by Pirkanmaa Hospital District's ethics committee (Decision R 14090M, October 13, 2014).

We sought to recruit women scheduled for elective or acute CS. Patients who underwent emergency CS or were unable to understand the Finnish language were excluded. Overall, 270 patients were randomized, 133 into the PCA group and 137 into the oral analgesia group. A flowchart of the recruitment process is shown in Fig. 1.

Patients were recruited to the study either in the outpatient clinic when the elective CS was arranged or in the delivery room when the decision for the acute CS was made. A written information letter and an oral explanation were given to the patients, and written consent was requested. A research nurse not participating in the treatment of the patients prepared envelopes including the information that assigned the patient to the IV PCA or oral group. A sealed opaque envelope was opened in the operating room by the operative nurse.

All patients were operated on under spinal anaesthesia. Spinal anaesthesia was performed using a 27 -gauge $\mathrm{BD}^{\mathrm{TM}}$ Quincke spinal needle at the L3-4 level. The patients were given intrathecal $0.5 \%$ hyperbaric bupivacaine $11 \mathrm{mg}$ and fentanyl $10 \mu \mathrm{g}$. Non-invasive arterial blood pressure was maintained above $-10 \%$ of the preoperative value using an intravenous crystalloid fluid infusion and boluses of intravenous phenylepinephrine $0.05 \mathrm{mg}$.

The patients had either a Pfannenstiel incision (263 patients) or a lower midline incision (3 patients in the IV PCA group and 4 in oral group).

Patients in both groups received extended-release oxycodone/naloxone 10/5 mg (OX/NAL) (oxycodone hydrochloride $10 \mathrm{mg}$ + naloxone hydrochloride $5 \mathrm{mg}$ ), ibuprofen $600 \mathrm{mg}$, and paracetamol $1 \mathrm{~g}$ orally $1 \mathrm{~h}$ after surgery. Thereafter, an OX/NAL dose was given every $12 \mathrm{~h}$, and ibuprofen and paracetamol were given every $8 \mathrm{~h}$.

In the IV PCA group, the patients received an intravenous PCA device (CADD Legacy PCA, Smiths Medical MD, Inc., St. Paul, MN, USA) with oxycodone $1 \mathrm{mg} / \mathrm{ml}$, using oxycodone bolus doses of $2 \mathrm{mg}$ and a lockout time of $10 \mathrm{~min}$. Patients were taught to use the pump in the operating theatre, and they were recommended to use it for at least $24 \mathrm{~h}$.

In the oral analgesia group, patients were given an oxycodone $5 \mathrm{mg}$ capsule upon request, the maximum dose being $60 \mathrm{mg}$ in $24 \mathrm{~h}$. The postoperative medication protocol is shown in Fig. 2.

Maternal pain scores and satisfaction with pain relief were asked and documented on the maternity ward. A numeric rating scale (NRS) was used. Pain and satisfaction were rated at 2, 4, 8, and $24 \mathrm{~h}$ after surgery. The pain scale ranged from 0 (=no pain) to 10 (= worst pain imaginable), and the satisfaction scale ranged from 0 (=completely dissatisfied) to 10 (= completely satisfied). Furthermore, the patient's subjective perception about gastrointestinal symptoms-such as nausea, vomiting, and abdominal distension-was asked at 4,8 , and $24 \mathrm{~h}$ after surgery and categorized into two groups: patients with no symptoms and patients with symptoms. The time point for mobilization, the first meal, and defecation was recorded as well.

The equianalgesic dose ratio between intravenous and oral oxycodone has been reported to be $1: 2$ [13, 14]; this ratio was therefore used to calculate the equianalgesic dose of oral oxycodone for the two groups.

\section{Statistical analysis}

Based on previous studies [15], the sample size calculations were made with the assumption that the standard deviations in pain scores would be 1.4, and a score difference between the groups of 0.5 would be regarded as clinically significant. 
Fig. 1 Distribution of patients during the study period

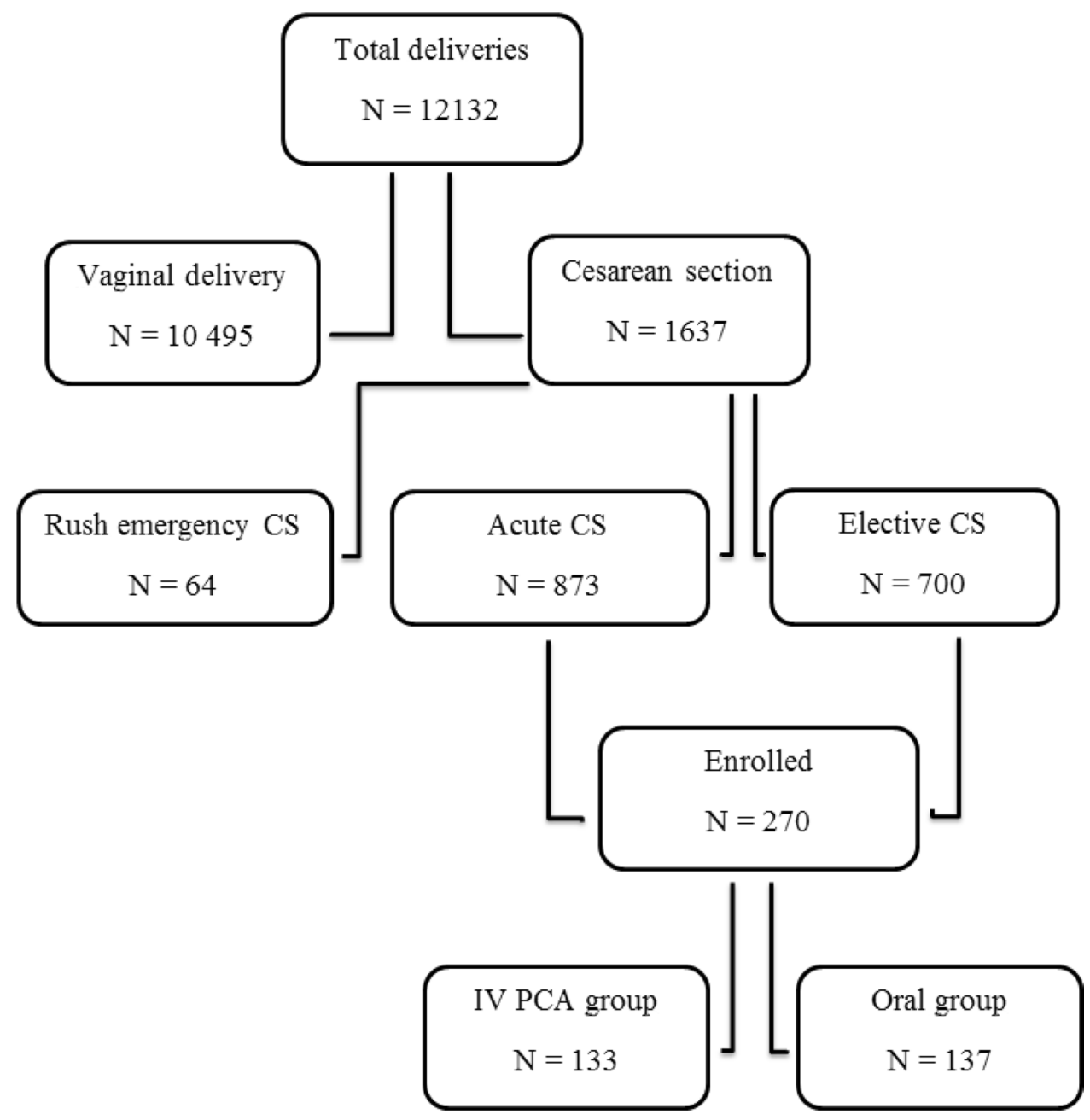

Two patients in the IV PCA group needed general anesthesia during $\mathrm{CS}$, one because of inadequate anesthesia and the other because of heavy bleeding during the operation. All other study patients were operated on under spinal anesthesia.

Five patients requested to have the IV PCA discontinued after a few hours' use because of side effects like nausea. Respectively, six patients in the oral analgesia group switched to an IV PCA later on because of pain. Epidural analgesia was used for one patient in the IV PCA group, and two patients in the IV PCA group were given extra oxycodone for intolerable pain. The mean usage time of the IV PCA was $19 \mathrm{~h}$ postoperatively.

There were no differences in pain at rest or satisfaction between the groups at $2,4,8$, and $24 \mathrm{~h}$ postoperatively, nor in pain when coughing at 2,4 , and $8 \mathrm{~h}$ postoperatively. At $24 \mathrm{~h}$, the NRS for pain at coughing was higher in the oral group ( $p=0.006$; Fig. 3a-c).

To determine the most discontented patients in the groups, the proportions of women with severe pain $(\mathrm{NRS} \geq 7)$ and dissatisfaction with pain treatment 


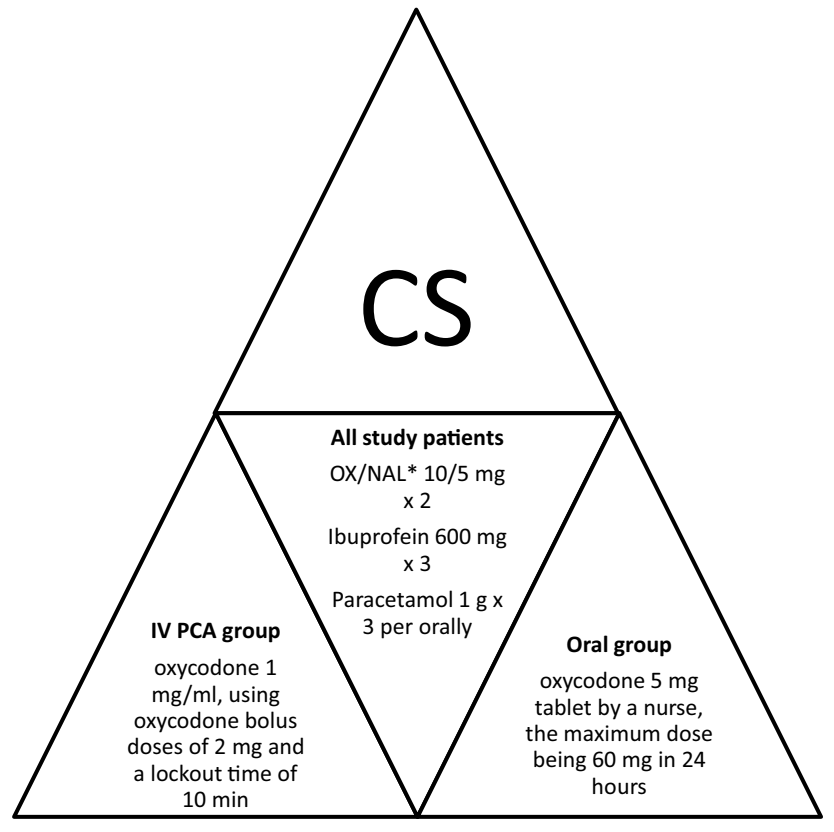

*OX/NAL $=$ extended-release oxycodone/naloxone tablet

Fig. 2 Postoperative medication protocol for the first $24 \mathrm{~h}$ after CS

$(\mathrm{NRS} \leq 3)$ were defined. At $24 \mathrm{~h}$ after $\mathrm{CS}$, five patients suffered severe pain at rest in the IV PCA group, compared to none in the oral group $(p=0.026)$. No other differences were found between the groups either in the experience of severe pain or in dissatisfaction with pain treatment (Table 2). Overall satisfaction (NRS $\geq 7$ ) for pain treatment was high: more than $77 \%$ were satisfied at every time point. There were no differences in severe pain or satisfaction between elective and acute CS patients.

Patients in the IV PCA group had more nausea at $4 \mathrm{~h}$ $(p=0.001)$ and vomited more often at $8 \mathrm{~h}(p=0.010)$. Otherwise, the groups did not differ in terms of gastrointestinal symptoms (Table 3). There was no difference in the recovery time of the groups (Table 4).

In the IV PCA group, the mean consumption of iv oxycodone was $58.2 \mathrm{mg}$ (SD 23.5), while in the oral group, the mean counted equianalgesic dose was $48.3 \mathrm{mg}$ (SD 11.8) during the first $24 \mathrm{~h}(p=0.003)$.

\section{Discussion}

In this prospective randomized study, we found that oral oxycodone was an equally effective and satisfactory means for postoperative analgesia after uncomplicated cesarean section as IV oxycodone administered by a patient-controlled device (PCA). However, nausea and vomiting were slightly more common in patients receiving IV PCA. Regarding postoperative pain scores and general satisfaction with pain relief, both methods were equally accepted by the patients. The results of our study are in line with those of Dieterich et al., who randomized 239 patients to receive either intravenous opioid piritramide (PCA) or oral oxycodone [16]. They used a visual pain scale (VAS) for pain assessment and found no differences between the groups. General satisfaction was also high, and Dieterich et al.'s results support the use of oral pain treatment after CS. Davis et al. also found that oral analgesia with oxycodone and acetaminophen after CS gave better pain relief with fewer side effects than an IV morphine PCA [17]. To our knowledge, no earlier studies comparing the per oral and IV PCA administration of oxycodone for post-cesarean pain and patient satisfaction exist.

Although alternative drugs, new combinations of existing drugs, and new applications of local anesthetics have been studied intensively, opioids are still required for post-cesarean analgesia [9]. Intravenous oxycodone is comparable to other IV opioids in terms of safety and efficacy [10], but even short-term opioid use by patients undergoing surgery can lead to chronic opioid use [18]. It is generally known
Table 1 Demographic data and parameters concerning the mother, pregnancy, and operation

\begin{tabular}{|c|c|c|c|c|}
\hline \multirow[t]{2}{*}{ Characteristic } & \multicolumn{2}{|c|}{ IV PCA group, $n=133$} & \multicolumn{2}{|c|}{ Oral group, $n=137$} \\
\hline & Median $/ n$ & $\operatorname{Min}-\max / \%$ & Median $/ n$ & $\operatorname{Min}-\max / \%$ \\
\hline Age (years) & 32 & $19-46$ & 33 & $20-43$ \\
\hline BMI $\left(\mathrm{kg} / \mathrm{m}^{2}\right)$ & 23.6 & $16.2-49.5$ & 24.6 & $17.5-52.6$ \\
\hline Primipara & 67 & 50.4 & 63 & 46.0 \\
\hline Gestational age at birth (days) & 274 & $208-295$ & 274 & $228-295$ \\
\hline Full-term $>37$ weeks & 121 & 91.0 & 128 & 93.4 \\
\hline Prior CS & 48 & 36.1 & 53 & 38.7 \\
\hline Elective CS & 107 & 80.5 & 114 & 83.2 \\
\hline Operation time (min) & 35 & $15-80$ & 33 & $15-75$ \\
\hline Bleeding (ml) & 500 & $80-4600$ & 450 & $100-3000$ \\
\hline
\end{tabular}

Values are expressed as the median or number of patients in the group and the parameter variables are expressed as the minimum to maximum or percentage in the group 
Fig. 3 a Pain scores at rest at different time points after the operation $(0=$ no pain, $10=$ the worst pain imaginable). b Pain scores when coughing at different time points after the operation $(0=$ no pain, $10=$ the worst pain imaginable). c Satisfaction with pain relief at different time points after the operation $(0=$ completely dissatisfied, $10=$ completely satisfied)

that rapidly affecting drugs increase this risk, therefore IV administration might be unfavorable. Moreover, the cost of the IV PCA, including medicine, infusion liquid and PCA device, is about twice as high as the oral medication.

It is challenging to treat postoperative pain efficiently without side effects. Especially after CS, one must consider not only the pain relief and wellbeing of the mother, but also active mother-child bonding and breast-feeding. It is also crucial to take into account the high risk of thromboembolism after CS [19]. Since delayed standing can negatively influence these goals, early postoperative mobilization and immediate removal of the urinary catheter are recommended after CS [20,21]. Thus, continuous epidural analgesia may not be favored, even if it might give excellent pain control. Intramuscular injection should be avoided, and transdermal opioids are appropriate only for persistent pain.

Self-administration with an IV PCA is the standard treatment in some hospitals, whereas oral administration is considered easier and practical in others. Compared to per oral administration, patients with an IV PCA can receive a drug therapy that is more individualized [22]. They receive immediate relief when needed, even for the slightest pain. However, unwanted side effects like nausea and drowsiness may delay recovery [17]. Per oral administration causes fewer side effects, but the pharmacological effect appears with a certain delay. Patients are also dependent on nurses, who are not always immediately available to deliver oral medication upon the patient's request. Nevertheless, in this study, the patients in both groups were equally satisfied with the pain relief.

As part of our study protocol, all patients received oral ibuprofen and paracetamol regularly every eight hours. Combined with the opioid, their safety and opioid-sparing effect have been documented in many studies. In the IV PCA group, patients used more oxycodone compared to the patients in the oral group. Regardless, there were no major differences between the groups in terms of the NRS, postoperative recovery, or satisfaction. The protocol of this study included per oral postoperative extended-release oxycodone, which entailed constant oxycodone content for all patients. By combining this with a non-steroidal antiinflammatory drug and paracetamol regularly, the need for short-acting extra oxycodone is considerably lower after surgery. This may be the reason why oxycodone administrated either by an IV PCA or orally ensured equal pain relief in our results. As is generally known, all patients usually
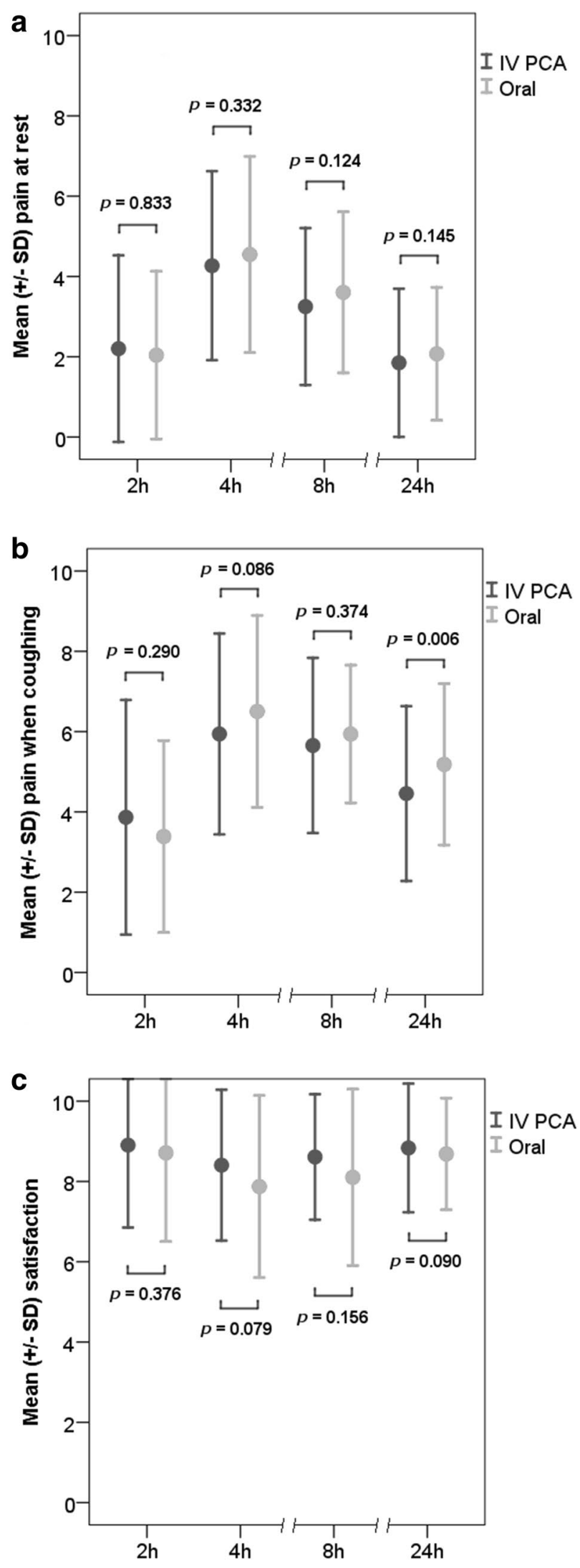
Table 2 Severe pain $(\mathrm{NRS} \geq 7)$ and dissatisfaction $(\mathrm{NRS} \leq 3)$ at different time points

\begin{tabular}{|c|c|c|c|c|c|}
\hline \multirow{2}{*}{$\begin{array}{l}\text { Characteristic } \\
\text { Time }\end{array}$} & \multicolumn{2}{|c|}{$\begin{array}{l}\text { IV PCA group, } \\
n=133\end{array}$} & \multicolumn{2}{|c|}{$\begin{array}{l}\text { Oral group, } \\
n=137\end{array}$} & \multirow[t]{2}{*}{$p$ value } \\
\hline & $\%$ & $n$ & $\%$ & $n$ & \\
\hline \multicolumn{6}{|c|}{ Severe pain at rest } \\
\hline $2 \mathrm{~h}$ & 8 & $10 / 119$ & 3 & $4 / 124$ & 0.083 \\
\hline $4 \mathrm{~h}$ & 21 & $26 / 123$ & 24 & $30 / 126$ & 0.614 \\
\hline $8 \mathrm{~h}$ & 8 & $9 / 120$ & 7 & $8 / 121$ & 0.788 \\
\hline $24 \mathrm{~h}$ & 5 & $5 / 106$ & 0 & $0 / 111$ & 0.026 \\
\hline \multicolumn{6}{|c|}{ Severe pain when coughing } \\
\hline $2 \mathrm{~h}$ & 20 & $24 / 119$ & 12 & $14 / 119$ & 0.077 \\
\hline $4 \mathrm{~h}$ & 43 & $51 / 119$ & 52 & $62 / 119$ & 0.153 \\
\hline $8 \mathrm{~h}$ & 38 & $43 / 113$ & 36 & $42 / 116$ & 0.772 \\
\hline $24 \mathrm{~h}$ & 17 & $18 / 103$ & 25 & $27 / 109$ & 0.194 \\
\hline \multicolumn{6}{|c|}{ Dissatisfaction with pain treatment } \\
\hline $2 \mathrm{~h}$ & 5 & $6 / 115$ & 5 & $6 / 118$ & 0.963 \\
\hline $4 \mathrm{~h}$ & 4 & $4 / 111$ & 6 & $7 / 119$ & 0.418 \\
\hline $8 \mathrm{~h}$ & 3 & $3 / 118$ & 8 & $9 / 117$ & 0.073 \\
\hline $24 \mathrm{~h}$ & 3 & $3 / 103$ & 1 & $1 / 108$ & 0.360 \\
\hline
\end{tabular}

Table 3 Gastrointestinal symptoms at different time points after CS in the two groups

\begin{tabular}{|c|c|c|c|c|c|}
\hline \multirow{2}{*}{$\begin{array}{l}\text { Characteristic } \\
\text { Time }\end{array}$} & \multicolumn{2}{|c|}{$\begin{array}{l}\text { IV PCA } \\
\text { group, } \\
n=133\end{array}$} & \multicolumn{2}{|c|}{$\begin{array}{l}\text { Oral group, } \\
n=137\end{array}$} & \multirow[t]{2}{*}{$p$ value } \\
\hline & $\%$ & $n$ & $\%$ & $n$ & \\
\hline Nausea 4 h & 16 & $19 / 121$ & 3 & $4 / 125$ & 0.001 \\
\hline Nausea $8 \mathrm{~h}$ & 9 & $11 / 121$ & 5 & $6 / 120$ & 0.215 \\
\hline Nausea 24 h & 5 & $5 / 105$ & 6 & $6 / 110$ & 0.818 \\
\hline Vomiting $4 \mathrm{~h}$ & 6 & $6 / 105$ & 2 & $2 / 109$ & 0.165 \\
\hline Vomiting $8 \mathrm{~h}$ & 10 & $11 / 108$ & 2 & $2 / 108$ & 0.010 \\
\hline Vomiting $24 \mathrm{~h}$ & 4 & $4 / 94$ & 0 & $0 / 97$ & 0.057 \\
\hline Abdominal distension $4 \mathrm{~h}$ & 18 & $21 / 117$ & 15 & $18 / 123$ & 0.487 \\
\hline Abdominal distension $8 \mathrm{~h}$ & 36 & $43 / 119$ & 42 & $49 / 119$ & 0.424 \\
\hline Abdominal distension $24 \mathrm{~h}$ & 70 & $73 / 105$ & 74 & $82 / 111$ & 0.478 \\
\hline
\end{tabular}

Table 4 Parameters concerning postoperative recovery expressed in hours

\begin{tabular}{lcrrrrr}
\hline Characteristic & \multicolumn{2}{l}{$\begin{array}{l}\text { IV PCA group, } \\
n=133\end{array}$} & & \multicolumn{2}{l}{ Oral group, $n=137$} & $p$ value \\
\cline { 2 - 3 } & Mean & SD & & Mean & SD & \\
\hline First meal & 7.8 & 5.5 & 7.0 & 4.6 & 0.336 \\
Mobilization & 17.4 & 7.4 & & 17.8 & 6.7 & 0.567 \\
Defecation & 60.1 & 18.3 & & 59.9 & 17.5 & 0.945 \\
\hline
\end{tabular}

benefit from participating in a clinical trial, regardless of the randomization.

When using an IV PCA, patients may have more nausea and drowsiness than with per oral analgesia [17], and this can negatively affect mobilization and baby care. Overall, nausea and vomiting after CS were rare events in our study compared to the study of Kim et al., where 127 patients had either an oxycodone or fentanyl IV PCA after a laparoscopic supracervical hysterectomy. Oxycodone was associated with superior analgesia, but those patients had a significantly higher incidence of side effects, such as postoperative nausea and vomiting, dizziness, and drowsiness [23]. In our study, over half the patients $(n=155 / 216)$ reported abdominal distension $24 \mathrm{~h}$ after surgery (no difference between study groups). Patients in the PCA group experienced more nausea at the 4-hour time point. Postoperative recovery was equal in both groups. The mean time of mobilization was $17 \mathrm{~h}$, which is an exceptionally long time compared to our 6-h recommendation. Postoperatively, the patients were given meals depending on the schedule of the ward, i.e., the time of operation determined the time that the meal was offered. The mean delay was $7 \mathrm{~h}$ postoperatively. The Cochrane review in 2014 found early postoperative feeding after major gynecological surgery to be safe and to enable the faster recovery of bowel function, a shorter hospital stay, and higher satisfaction [24].

The strengths of this study include the large, randomized, non-selected material, and the comparison of two treatments that are normal in clinical use. A potential weakness of this study was the relatively low recruitment percentage of acute CS patients, which was $19.5 \%$ (26/133) in the IV PCA group and $16.8 \%$ (23/137) in the oral group. The difference between pain relief and postoperative recovery between the elective and acute CS could not be reliably estimated.

In conclusion, the results of this study show that oral oxycodone is an effective and satisfactory method for postoperative analgesia after cesarean section. IV oxycodone administered with the use of a PCA did not offer clinically significant benefits. Both methods were generally well tolerated, and pain relief was good with both methods. Early nausea was less common with oral medication. Therefore, our findings do not support the routine use of a IV oxycodone PCA in this patient group.

Author contributions KKM: protocol development, data collection, data analysis, manuscript writing. OP: protocol development, manuscript writing. JU: protocol development, manuscript writing. AY-H: manuscript writing. SP: manuscript editing. $\mathrm{MH}$ : data analysis, manuscript editing.

\section{Compliance with ethical standards}

Conflict of interest We declare that we have no conflict of interest. 
Ethical approval All procedures performed in studies involving human participants were in accordance with the ethical standards of the institutional and/or national research committee and with the 1964 Helsinki Declaration and its later amendments or comparable ethical standards.

Informed consent Informed consent was obtained from all individual participants included in the study.

Open Access This article is distributed under the terms of the Creative Commons Attribution 4.0 International License (http://creativeco mmons.org/licenses/by/4.0/), which permits unrestricted use, distribution, and reproduction in any medium, provided you give appropriate credit to the original author(s) and the source, provide a link to the Creative Commons license, and indicate if changes were made.

\section{References}

1. Molina G, Weiser TG, Lipsitz SR et al (2015) Relationship between cesarean delivery rate and maternal and neonatal mortality. JAMA 314(21):2263-2270. https://doi.org/10.1001/ jama.2015.15553

2. Keag OE, Norman JE, Stock SJ (2018) Long-term risks and benefits associated with cesarean delivery for mother, baby, and subsequent pregnancies: systematic review and meta-analysis. PLoS Med 15(1):e1002494. https://doi.org/10.1371/journal.pmed.10024 94

3. Lavender T, Hofmeyr GJ, Neilson JP, Kingdon C, Gyte GML (2012) Caesarean section for non-medical reasons at term. Cochrane Database Syst Rev 3:CD004660

4. Perinatal statistics-parturients, deliveries and newborns 2017. Statistical report 38/2018. National Institute for Health and Welfare (THL). https://thl.fi/en/web/thlfi-en/statistics/statistics -by-topic/sexual-and-reproductive-health/parturientsdeliverie s-and-births/perinatal-statistics-parturients-delivers-and-newbo rns. Accessed 15 Aug 2019

5. Gerbershagen HJ, Aduckathil S, van Wijck AJ, Peelen LM, Kalkman CJ, Meissner W (2013) Pain intensity on the first day after surgery: a prospective cohort study comparing 179 surgical procedures. Anesthesiology 118(4):934-944

6. Taneja B, Kerai S, Saxena K (2017) Post-caesarean analgesia: what is new? (review article). Indian J Anaesth 61(3):200-214

7. Katz P, Takyar S, Palmer P, Liedgens H (2017) Sublingual, transdermal and intravenous patient-controlled analgesia for acute postoperative pain: systematic literature review and mixed treatment comparison. Curr Med Res Opin 33(5):899-910

8. Carvalho B, Butwick AJ (2017) Postcesarean delivery analgesia. Best Pract Res Clin Anaesthesiol 31(1):69-79

9. Schyns-van den Berg AM, Huisjes A, Stolker RJ (2015) Postcaesarean section analgesia: are opioids still required? Curr Opin Anaesthesiol 28(3):267-274

10. Pergolizzi JV, SeowChoen F, Wexner SD, Zampogna G, Raffa RB, Taylor R (2016) Perspectives on intravenous oxycodone for control of postoperative pain. Pain Pract 16(7):924-934
11. Olkkola KT, Kontinen VK, Saari TI, Kalso EA (2013) Does the pharmacology of oxycodone justify its increasing use as an analgesic? Trends Pharmacol Sci 34(4):206-214

12. Cheung CW, Ching Wong SS, Qiu Q, Wang X (2017) Oral oxycodone for acute postoperative pain: a review of clinical trials. Pain Phys 20(2S):SE52

13. Kalso E (2005) Oxycodone. J Pain Symptom Manage 29(5 Suppl): 47

14. Poyhia R, Vainio A, Kalso E (1993) A review of oxycodone's clinical pharmacokinetics and pharmacodynamics. J Pain Symptom Manag 8(2):63-67

15. Ebneshahidi A, Akbari M, Heshmati B (2012) Patient-controlled versus nurse-controlled post-operative analgesia after caesarean section. Adv Biomed Res 1:9175.94428 (Epub 2012 Mar 28)

16. Dieterich M, Müller-Jordan K, Stubert J, Kundt G, Wagner K, Gerber B (2012) Pain management after cesarean: a randomized controlled trial of oxycodone versus intravenous piritramide. Arch Gynecol Obstet 286(4):859-865

17. Davis KM, Esposito MA, Meyer BA (2006) Oral analgesia compared with intravenous patient-controlled analgesia for pain after cesarean delivery: a randomized controlled trial. Obstet Gynecol 194(4):967-971

18. Sun EC, Darnall BD, Baker LC, Mackey S (2016) Incidence of and risk factors for chronic opioid use among opioid-naive patients in the postoperative period. JAMA Intern Med 176(9):1286-1293. https://doi.org/10.1001/jamainternmed.2016.3298

19. Blondon M, Casini A, Hoppe KK, Boehlen F, Righini M, Smith NL (2016) Risks of venous thromboembolism after cesarean sections: a meta-analysis. Chest 150(3):572-596

20. ACOG committee opinion no. 750 (2018) Obstet Gynecol 132(3): 130

21. El-Mazny A, El-Sharkawy M, Hassan A (2014) A prospective randomized clinical trial comparing immediate versus delayed removal of urinary catheter following elective cesarean section. Eur J Obstet Gynecol Reprod Biol 181:111-114

22. Pettersson P, Anjou Lindskog E, Owall A (2000) Patient-controlled versus nurse-controlled pain treatment after coronary artery bypass surgery. Acta Anaesthesiol Scand 44(1):43-47

23. Kim NS, Lee JS, Park SY et al (2017) Oxycodone versus fentanyl for intravenous patient-controlled analgesia after laparoscopic supracervical hysterectomy: a prospective, randomized, doubleblind study. Medicine (Baltimore) 96(10):e6286

24. Charoenkwan K, Matovinovic E (2014) Early versus delayed oral fluids and food for reducing complications after major abdominal gynaecologic surgery. Cochrane Database Syst Rev 12:CD004508

Publisher's Note Springer Nature remains neutral with regard to jurisdictional claims in published maps and institutional affiliations. 